\section{Evaluation of a loop-mediated isothermal amplification method for the detection of Listeria monocytogenes in dairy food}

Erica Tirloni, ${ }^{1}$ Cristian Bernardi, ${ }^{1}$ Sandro Drago, ${ }^{2}$ Giuseppe Stampone, ${ }^{2}$ Francesco Pomilio, ${ }^{3}$ Patrizia Cattaneo, ${ }^{1}$ Simone Stella ${ }^{1}$

${ }^{1}$ Department of Health, Animal Science and Food Safety, University of Milan; ${ }^{2}$ Enbiotech srl, Palermo; ${ }^{3}$ Institute for Experimental Veterinary Medicine of Abruzzo and Molise G. Caporale, National Reference Laboratory for Listeria monocytogenes

\section{Abstract}

Objective of the present study was to test the performances of a loop-mediated isothermal amplification (LAMP)-based method for the detection of Listeria monocytogenes, with particular focus on the dairy products. The specificity of the method was evaluated on 42 different Listeria spp. strains from collections, food and environmental samples. $100 \%$ (32 of 32) of the $L$. monocytogenes strains were correctly recognised, and none of other 10 Listeria spp. strains was misidentified. The sensitivity was evaluated on four $L$. monocytogenes strains from different sources. The instrument was able to detect 10-400 CFU/mL. The ability to detect low initial numbers of L. monocytogenes (0.3$0.7 \mathrm{Log} \mathrm{CFU} / \mathrm{g}$ ) was also evaluated, in duplicate, in pasteurised milk (whole and skimmed) and dairy samples (fresh ricotta, crescenza, mascarpone, mozzarella, cottage cheese, cream cheese, taleggio, gorgonzola). The analysis was performed after 18, 24 and $48 \mathrm{~h}$ of incubation, and was coupled with the count of L. monocytogenes in the broth. Microbial loads were insufficient to achieve a positive result after 18 and $24 \mathrm{~h}$ in most of the samples; after $48 \mathrm{~h}$, all the products, except taleggio and one gorgonzola sample, were identified as positive; the sensitivity of the method when applied to contaminated dairy foods was about $5 \mathrm{Log}$ CFU/g. The LAMP method tested can be considered a very useful tool, as it is a costeffective and easy-functioning method. The preliminary data obtained should be confirmed with a validation process taking into account different food typologies.

\section{Introduction}

Along the food supply chain, food safety hazard may enter at several stages: although safety control programs are currently applied, microbiological contaminations often occur. Rapid Alert System for Food and Feed database (RASFF) reported a total of 243 notifications due to microorganisms on foodstuffs in the period January-May $2017,95 \%$ of which related to pathogenic microorganisms like Listeria monocytogenes, Escherichia coli, Salmonella spp., Staphylococcus aureus and Bacillus cereus. A total of 28 notifications $(12.1 \%$ of those caused by pathogenic bacteria) were related to the presence of $L$. monocytogenes in a wide and heterogeneous group of Ready To Eat (RTE) foods including chilled smoked salmon and halibut, chilled beef and ham, cheeses (soft or blue) and multi-ingredients foods (cakes).

Listeriosis is a relatively rare foodborne disease with more than 2200 confirmed cases in the EU in 2015. L. monocytogenes is a microorganism of particular concern due to high fatality (15-18\%) and hospitalisation rates (97-99\%), if compared to other microbial pathogens (EFSA, 2015, 2016). Moreover, the microorganism is widely distributed in the food processing plants, and it possesses the peculiarity to grow in a wide range of temperature (from 0 to $45^{\circ} \mathrm{C}$ ) and to resist to many environmental conditions, such as high salinity or acidity (Walker et al., 1990). RTE (including dairy products) resulted to be in recent times often associated to outbreaks and sporadic cases of listeriosis (WHO-FAO, 2004; EFSA, 2016).

Among dairy products, fresh cheeses constitute suitable growth media for several spoilage and pathogenic bacteria and have worldwide been associated with foodborne microbial outbreaks, with an important role of L. monocytogenes (Zottola and Smith, 1991). The infections can be severe or fatal for the consumers; moreover the presence of the pathogen could have a serious negative impact on the turnover of Italian dairy industry in terms of economic losses and negative advertising. L. monocytogenes can be likely present in raw materials (such as raw milk), but can enter the production chain during the process or contaminate the final product during ripening, due to postcontamination or cross-contaminations events. Furthermore, L. monocytogenes is often isolated from cheese making environment such as floors, drains and equipment; moreover this pathogen persist for many years in several niches in the dairy processing plants (Unnerstad et al., 1996; Spanu et al., 2015). Traditional pathogen detection methods are based on microbiological cul-
Correspondence: Erica Tirloni, Department of Health, Animal Science and Food Safety, University of Milan, Via Celoria 10, 20133 Milan, Italy.

Tel: +39.02 .50317855 , Fax: +39.02 .50317870$

E-mail: erica.tirloni@unimi.it

Key words: LAMP, Listeria monocytogenes, RTE dairy food, limit of detection.

Conflict of interest: the authors declare no potential conflict of interest.

Received for publication: 7 July 2017

Revision received: 10 October 2017

Accepted for publication: 13 October 2017.

This work is licensed under a Creative Commons Attribution-NonCommercial 4.0 International License (CC BY-NC 4.0).

CCopyright E. Tirloni et al., 2017

Licensee PAGEPress, Italy

Italian Journal of Food Safety 2017; 6:6890

doi:10.4081/ijfs.2017.6890

ture techniques that, despite the consistency of the results obtained, sometimes had shown some weaknesses especially in terms of selective cultivation of some pathogens (Kim et al., 2014); moreover, culture methods need long technical execution times and incubation periods to obtain the final results. Thus, a rapid, accurate and sensitive diagnostic test for the presence and quantification of potential pathogenic microorganisms like L. monocytogenes in food may be desirable and could be extremely useful especially for an application in HACCP control plans.

Loop-mediated isothermal amplification (LAMP) is a relatively new real time DNA-amplification technique, firstly described by Notomi et al. (2000). The main advantage of LAMP if compared to PCR is that it does not need complex thermal cyclers and results could be obtained within few hours (Mori et al., 2001; Nagamine et al., 2002). Thus, in recent years LAMP has been deeply studied and applied as an easy, rapid and cost-effective field-friendly diagnostic tool for the identification of several potential pathogenic bacteria and parasites like Salmonella spp., Listeria monocytogenes and Anisakis spp. (Hara-Kudo et al., 2005; Wang et al., 2010; Tang et al., 2011; Wan et al., 2012; Tirloni et al., 2017). Anyway, few studies focused on the reliability and suitability of LAMP technology when applied to complex and structured matrixes (Hara-Kudo et al., 2007; Zhang et al., 2012).

The objective of the present study was to test the performances in terms of speci- 
ficity and sensitivity of a LAMP-based method for the evaluation of the presence of L. monocytogenes with particular focus on the detection of the pathogen in different dairy products.

\section{Materials and Methods}

\section{Evaluation of the specificity of the method}

A total of 42 Listeria spp. strains were considered as reported in Table 1. Part of these strains, belonging to the European Union Reference Laboratory (EURL) set, were supplied by the National Reference Laboratory for Listeria monocytogenes (strains from 1 to 25), while the other strains belong to the internal laboratory collection and were isolated from food of animal origin (strains 26 to 31 ) or environmental samples (strains from 34 to 42). Also two American Type Culture Collection (ATCC) strains were used (strains 32 and 33). Strain stocks were kept at $-80^{\circ} \mathrm{C}$ until a loop $(\sim 10$ $\mu \mathrm{L})$ was transferred in Tryptic Soy broth tubes, subsequently incubated at $37^{\circ} \mathrm{C}$ for $48 \mathrm{~h}$. At the end of the incubation period, cells were harvested in exponential growth phase, defined as a relative change in optical density (OD) of $0.05-0.2$ at $540 \mathrm{~nm}$ (6320D spectrophotometer; Jenway, Staffordshire, UK). Cell concentration of each bacterial strain suspension was calculated by counting under a phase-contrast microscopy (BA 310, Motic, Barcelona, E) and, if necessary, was diluted in sterile saline water $(0.85 \% \mathrm{NaCl})$, to obtain a concentration of $8 \mathrm{Log} \mathrm{CFU} / \mathrm{mL}$.

\section{Description and operative protocol of loop-mediated isothermal amplifi- cation diagnostic test for $L$. monocy- togenes}

For the tests, the system ICGENE mini (Enbiotech, Palermo, Italy) was evaluated. The system is based on LAMP technology, and is composed by a fluorescence amplifier, able to detect the fluorescence emitted by the samples (12 samples can be analysed simultaneously), and a device that functions with Radio Frequency Identification technology (RFID), able to guide step by step all along the activities and to perform the automatic interpretation of the data. In this trial, specific kits named ICGENE Listeria monocytogenes developed for the detection of Listeria monocytogenes were obtained and tested. The operative protocol of the instrument, provided by the producer, is described below.

\section{DNA extraction}

One $\mathrm{mL}$ of each broth culture was collected and put into appropriate tubes, which were subsequently centrifuged at $8000 \mathrm{rpm}$ for 5 minutes. Supernatants were discarded and pellet was re-suspended with extraction buffer and immediately homogenised by vortex to allow the extraction of nucleic acid by chemical lysis. The homogenate was incubated at room temperature for at least 10 minutes and newly centrifuged in the same conditions. The supernatant obtained was used as the template.

\section{Amplification (loop-mediated isothermal amplification) phase}

Three $\mu \mathrm{L}$ of the template were put into primer mix tubes with $22 \mu \mathrm{L}$ of LAMP mix (Enbiotech, Palermo, Italy) containing a lyophilic formulation primer and a master mix with reagents useful to carry out the test (enzyme, Magnesium chloride, nucleotides and reaction buffers) and $30 \mu \mathrm{L}$ of mineral oil. Finally, tubes are vortexed in order to obtain a homogenous solution, and inserted into the instrument. The reaction amplification consists of 60 minutes at $65^{\circ} \mathrm{C}$; the positivity is revealed graphically by the sigmoid curve produced by the instrument. For food products, at the end of the amplification stage and before collecting $3 \mu \mathrm{L}$ to put into the primer mix, template was diluted 1:5 with extraction buffer with the aim to avoid the interference of the fat component.

\section{Evaluation of the sensitivity of the method}

To evaluate the sensitivity of the LAMP method, four representative Listeria monocytogenes strains taken from the EURL set, isolated from different sources (strain 045: meat; 099: seafood; 118: dairy; 049: other food), were selected. Strain suspensions were prepared as described above, obtaining a cell concentration of $8 \mathrm{Log} C F U / \mathrm{mL}$. Serial 10-fold dilutions were prepared; all the broths and their respective dilutions were tested following the protocols reported above to highlight the limit of detection of the instrument. Contemporary, to enumerate the bacterial concentrations, all the dilutions were plated onto the selective media for the enumeration of the pathogen, Rapid L'mono (Generon, San Prospero, Italy) that was afterwards incubated at $37^{\circ} \mathrm{C}$ for $48 \mathrm{~h}$.

\section{Application to dairy samples}

Ten different dairy products were chosen, covering different chemical-physical and structural characteristics. The following dairy products were considered: pasteurised whole and skim milk were considered for the unstructured matrices, for the low fat percentage (3.6 and $1.6 \%$, respectively) and the limited presence of natural microflora.
Industrial hot-sealed fresh ricotta was chosen for its soft structure, the absence of resident bacteria and its permissive intrinsic characteristics $(\mathrm{pH}$ 6-6.5, high moisture content). Mozzarella cheese was included as it is characterised by high moisture and permissive $\mathrm{pH}$ (usually 5,5-6,5). Moreover, other low-structured cheeses with different fat content were selected: crescenza cheese $(\sim 25 \%)$, mascarpone cheese $(\sim 42 \%)$, cream cheese $(\sim 27.5 \%)$ and cottage cheese $(\sim 4.5 \%)$. Finally, ripened cheeses (e.g. taleggio and gorgonzola cheeses) with high fat content $(\sim 26-27 \%)$ and the presence of natural microflora (lactic acid bacteria and moulds) were selected. Specific challenge tests were carried out to determine whether LAMP technology was able to detect $L$. monocytogenes in real-food samples contaminated with low (realistic) concentrations of the pathogen. Growth of L. monocytogenes was determined in 20 independent challenge tests, 2 for each selected foodstuff.

Twenty five $\mathrm{g}$ of each product were inoculated with $0.3-0.7 \mathrm{Log} \mathrm{CFU} / \mathrm{g}$ of $L$. monocytogenes strain suspension (strain 118 of EURL set, isolated from a dairy product), prepared as above described for $L$. monocytogenes strains and 10-fold diluted with $225 \mathrm{ml}$ of Half Fraser Broth (Oxoid, Basingstoke, UK); the samples were then incubated at $30^{\circ} \mathrm{C}$ for $24 \mathrm{~h}$. LAMP analyses were carried out from samples at the moment of inoculation and after 18, 24 and $48 \mathrm{~h}$ of incubation, to evaluate if bacterial concentrations of L. monocytogenes could be detected by the device. At the meantime, L. monocytogenes was counted by spread plating onto Rapid L'mono.

\section{Results}

\section{Evaluation of specificity}

The results obtained from the evaluation of specificity of LAMP technology showed that $100 \%$ of Listeria monocytogenes strains selected for the tests were correctly identified (32/32 strains) (Table 1). None of other 10 Listeria spp. strains tested (including L. innocua, L. grayi, L. seeligeri, L. ivanovii and L. welshimeri) was recognised by the system.

\section{Evaluation of sensitivity}

For strain 045, amplification curves were revealed starting from loads of $4 \times 10^{2}$ $\mathrm{UFC} / \mathrm{mL}$ with a detection time between 15 and 40 minutes from the beginning of the amplification (Figure 1). The presence of $L$. monocytogenes was detected when the broth was less diluted, thus for higher con- 
centrations. Considering 049 strain, amplification curves were revealed starting from loads of $50 \mathrm{UFC} / \mathrm{mL}$ with a detection time between 15 and more than 50 minutes from the beginning of the amplification stage. As it can be seen in Figure 1, in this case the detection time was evidently correlated with the dilution of the broths for all the dilution series. For strain 099, amplification curves were revealed starting from loads from $10 \mathrm{UFC} / \mathrm{mL}$ (Figure 2). The detection time observed was between 25 minutes (for the broth diluted $10^{-1}$, line green) till more than 45 minutes (broth diluted $10^{-5}$, red line) from the beginning of the amplification. For strain 118, amplification curves were revealed from a broth load of $10 \mathrm{UFC} / \mathrm{mL}$ (Figure 2). The detection time observed was between 25 minutes and 35 minutes from the beginning of the amplification.

\section{Application of loop-mediated isothermal amplification to dairy samples}

The tests performed on milk and dairy products samples were intended to reveal the potential application of the LAMP technology in routinely analytical activity, such as in the presence of different chemicalphysical and microbiological conditions (fat, $\mathrm{pH}$, natural microflora, etc.) and low $L$. monocytogenes contamination. An eventual application of short incubation time was also evaluated, by testing samples incubated for different periods (18, 24 and $48 \mathrm{~h})$. At T0, as expected, the presence of the pathogen was not revealed in any of the samples, due to the low inoculation level (7 CFU/250 g, corresponding to $0.03 \mathrm{CFU} / \mathrm{mL}$ of broth), considering that the LAMP analysis is performed on a $1 \mathrm{~mL}$ broth share. After $18 \mathrm{~h}$ of incubation, an evident growth of L. monocytogenes in Half Fraser broth was detected, with microbial counts ranging among $1.0 \mathrm{Log} \mathrm{CFU} / \mathrm{mL}$ (gorgonzola cheese B) and $3.4 \mathrm{Log} \mathrm{CFU} / \mathrm{mL}$ (whole milk $A$ and skim milk A) (Table 2), but these microbial loads were not sufficient to be revealed by the method. After $24 \mathrm{~h}, L$. monocytogenes grew up to loads above to 4 $\mathrm{Log} \mathrm{CFU} / \mathrm{mL}$ in all the samples, with the only exception of the two taleggio cheeses, where a mean count of $2.6 \mathrm{Log} \mathrm{CFU} / \mathrm{mL}$ was detected, and one gorgonzola cheese sample (count $=2.3 \mathrm{Log} \mathrm{CFU} / \mathrm{mL}$ ). After $48 \mathrm{~h}$ of incubation, in almost all the tested dairy foods, L. monocytogenes was identified by the LAMP method (Figure 3). The microbial loads enumerated were mainly above $8 \log \mathrm{cfu} / \mathrm{mL}$, with the only exception of gorgonzola cheese (a sample with count close to $6 \mathrm{Log} \mathrm{CFU} / \mathrm{mL}$ and the other near 3 Log CFU/mL) and taleggio cheese samples (both with count close to $3 \mathrm{Log} \mathrm{cfu} / \mathrm{mL}$ ): the
LAMP method confirmed the weak growth in these substrates, giving negative results. The amplification curves started growing in a variable period, ranging mainly from 30 to 40 minutes, values that were delayed, as expected, if compared with those obtained

Table 1. Listeria spp. strains selected for the tests and relative results from loop-mediated isothermal amplification detection method.

\begin{tabular}{|c|c|c|c|}
\hline Strain & Identification & Source & Results \\
\hline 1 & L. monocytogenes & Meat & + \\
\hline 2 & L. monocytogenes & Meat & + \\
\hline 3 & L. monocytogenes & Food different from meat, seafood and dairy & + \\
\hline 4 & L. monocytogenes & Food different from meat, seafood and dairy & + \\
\hline 5 & L. monocytogenes & Food different from meat, seafood and dairy & + \\
\hline 6 & L. monocytogenes & Food different from meat, seafood and dairy & + \\
\hline 7 & L. monocytogenes & Food different from meat, seafood and dairy & + \\
\hline 8 & L. monocytogenes & Food different from meat, seafood and dairy & + \\
\hline 9 & L. monocytogenes & Dairy product & + \\
\hline 10 & L. monocytogenes & Seafood & + \\
\hline 11 & L. monocytogenes & Meat & + \\
\hline 12 & L. monocytogenes & Meat & + \\
\hline 13 & L. monocytogenes & Dairy product & + \\
\hline 14 & L. monocytogenes & Dairy product & + \\
\hline 15 & L. monocytogenes & Seafood & + \\
\hline 16 & L. monocytogenes & Seafood & + \\
\hline 17 & L. monocytogenes & Seafood & + \\
\hline 18 & L. monocytogenes & Seafood & + \\
\hline 19 & L. monocytogenes & Seafood & + \\
\hline 20 & L. monocytogenes & Seafood & + \\
\hline 21 & L. monocytogenes & Dairy product & + \\
\hline 22 & L. monocytogenes & Dairy product & + \\
\hline 23 & L. monocytogenes & Seafood & + \\
\hline 24 & L. monocytogenes & Meat & + \\
\hline 25 & L. monocytogenes & Dairy product & + \\
\hline 26 & L. monocytogenes & Taleggio cheese & + \\
\hline 27 & L. monocytogenes & Salami & + \\
\hline 28 & L. monocytogenes & Smoked cooked ham & + \\
\hline 29 & L. monocytogenes & Raw ham & + \\
\hline 30 & L. monocytogenes & Shrimp mayonnaise cocktail & + \\
\hline 31 & L. monocytogenes & Sandwich & + \\
\hline 32 & L. monocytogenes & ATCC & + \\
\hline 33 & L. innocua & ATCC & - \\
\hline 34 & L. ivanovii & Environmental sample & - \\
\hline 35 & L. ivanovii & Environmental sample & - \\
\hline 36 & L. grayi & Environmental sample & - \\
\hline 37 & L. welshimeri & Environmental sample & - \\
\hline 38 & L. seeligeri & Environmental sample & - \\
\hline 39 & L. innocua & Environmental sample & - \\
\hline 40 & L. innocua & Environmental sample & - \\
\hline 41 & L. welshimeri & Environmental sample & - \\
\hline 42 & L. grayi & Environmental sample & - \\
\hline
\end{tabular}

ATCC, American Type Culture Collection. 
in broth cultures with similar bacterial concentrations (Figures 1-3).

\section{Discussion}

In recent years, consumer needs are focusing more and more in public health matching also the overwhelming need expressed by food industries and competent governmental authorities to determine as soon as possible the eventual presence of $L$. monocytogenes in foods.

In this study, a new LAMP method was evaluated firstly in terms of specificity by testing broth cultures. The method evaluated was able to properly identify all L. monocytogenes strains tested, while no Listeria spp. strain was misidentified: these findings suggested that hly $A$ gene used as target to design the primers was adequate to detect specifically the pathogen.

Secondly, the test was assessed in terms of sensitivity resulting able to detect $L$. monocytogenes loads from 10 to 400 $\mathrm{UFC} / \mathrm{mL}$; the sensitivity of this method was in agreement with values reported in literature for comparable instruments based on the same technique, also if a certain variability was shown by different authors. Cho et al. (2014) reported a LOD equal to 2.22 CFU/mL under optimal conditions, while Wang et al. (2012) reported a LOD of $10.000 \mathrm{CFU} / \mathrm{mL}$ in pure L. monocytogenes cultures. Tang et al. (2011) stated a LOD of 2 UFC/reaction (corresponding to 20 $\mathrm{CFU} / \mathrm{mL}$ ). Considering the time required for the detection, broths with higher $L$. monocytogenes concentrations gave constantly a positive result within half an hour; in some cases (e.g. strain 049), a relation between the microbial concentration and amplification time was clearly recognised; generally, in the presence of low bacterial concentrations, a higher variability was observed, reaching, in some cases, times near the whole amplification course (1 hour).

Finally, the LAMP assay was applied to dairy products mimicking a low starting contamination and a subsequent subculturation in a selective broth, according to the ISO technique for the detection of the pathogen. As expected, the detection limit assessed in dairy substrates was higher if compared to broths. After $18 \mathrm{~h}$ of incubation of the products voluntarily contaminated with $L$. monocytogenes and enriched in selective broths, none of the samples was identified, while after $24 \mathrm{~h}$, only few samples resulted positive after LAMP analyses. This may be due to the higher number of hurdles present in the matrix of these dairy products such as natural microflora present
A

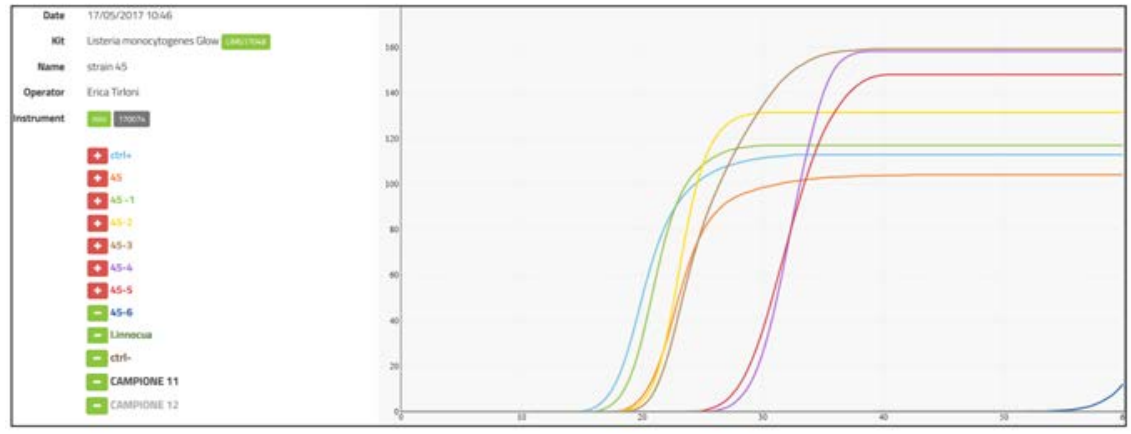

B

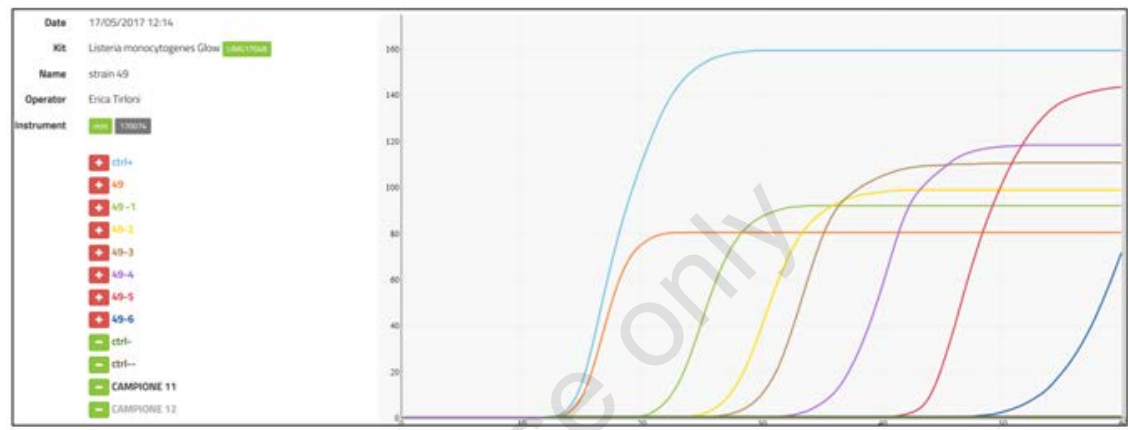

Figure 1. Sensitivity test results of strain 45 (A) and 49 (B).

A

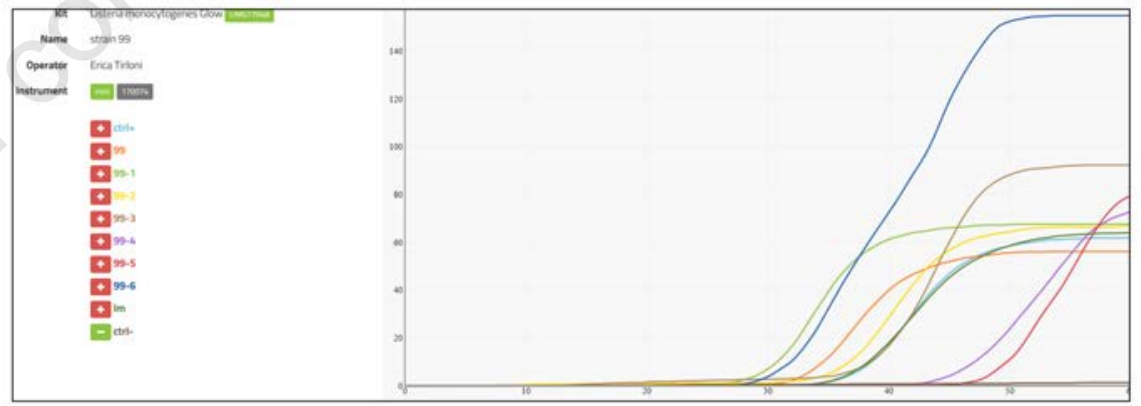

B

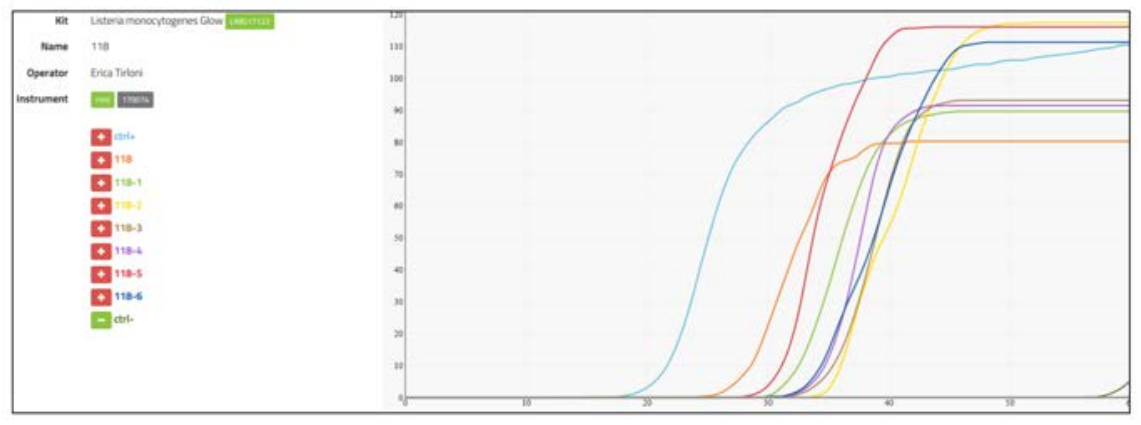

Figure 2. Sensitivity test results of strain 99 (A) and 118 (B). 
or chemical-physical conditions. Milk, ricotta and cream cheese as previously reported (Zottola and Smith, 1991), resulted to be an optimal substrate for Listeria growth, as no natural microflora was able to exert an inhibitory activity. In some of the matrixes considered, like mozzarella, crescenza and cottage cheese, although the presence of natural lactic microflora, the pathogen showed a growth, reaching mean loads after $24 \mathrm{~h}$ of incubation around 4-5 $\log \mathrm{CFU} / \mathrm{mL}$, that in any case were not sufficient to be recorded by the LAMP device. The impossibility to reveal the presence of L. monocytogenes after $24 \mathrm{~h}$ was likely due to a kind of interference of the food substrate, as the pathogen was easily detected also at lower concentrations in inoculated broths.

In the present study, the LAMP method tested resulted in the identification of the majority of the positive samples in $48 \mathrm{~h}$, when loads were close to $5 \mathrm{Log} \mathrm{CFU} / \mathrm{g}$; the time required for reaching this value depended on the incubation conditions and, as shown by our results, by the food matrix.

Generally, routine culture-based methods remain the more frequently used tech-
A

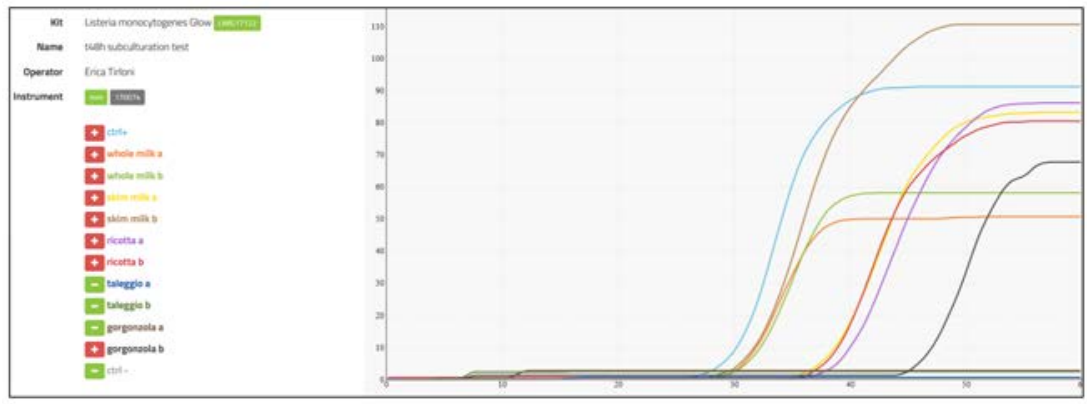

B

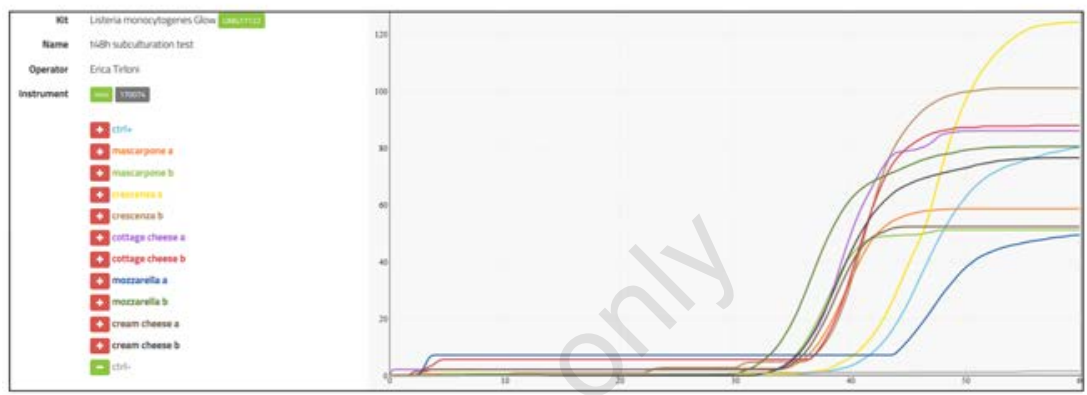

Figure 3. Amplification curves obtained from loop-mediated isothermal amplification identification after $48 \mathrm{~h}$ of subculturation.

Table 2. Results obtained by loop-mediated isothermal amplification detection method after 18, 24 and $48 \mathrm{~h}$ of incubation and relative microbial counts.

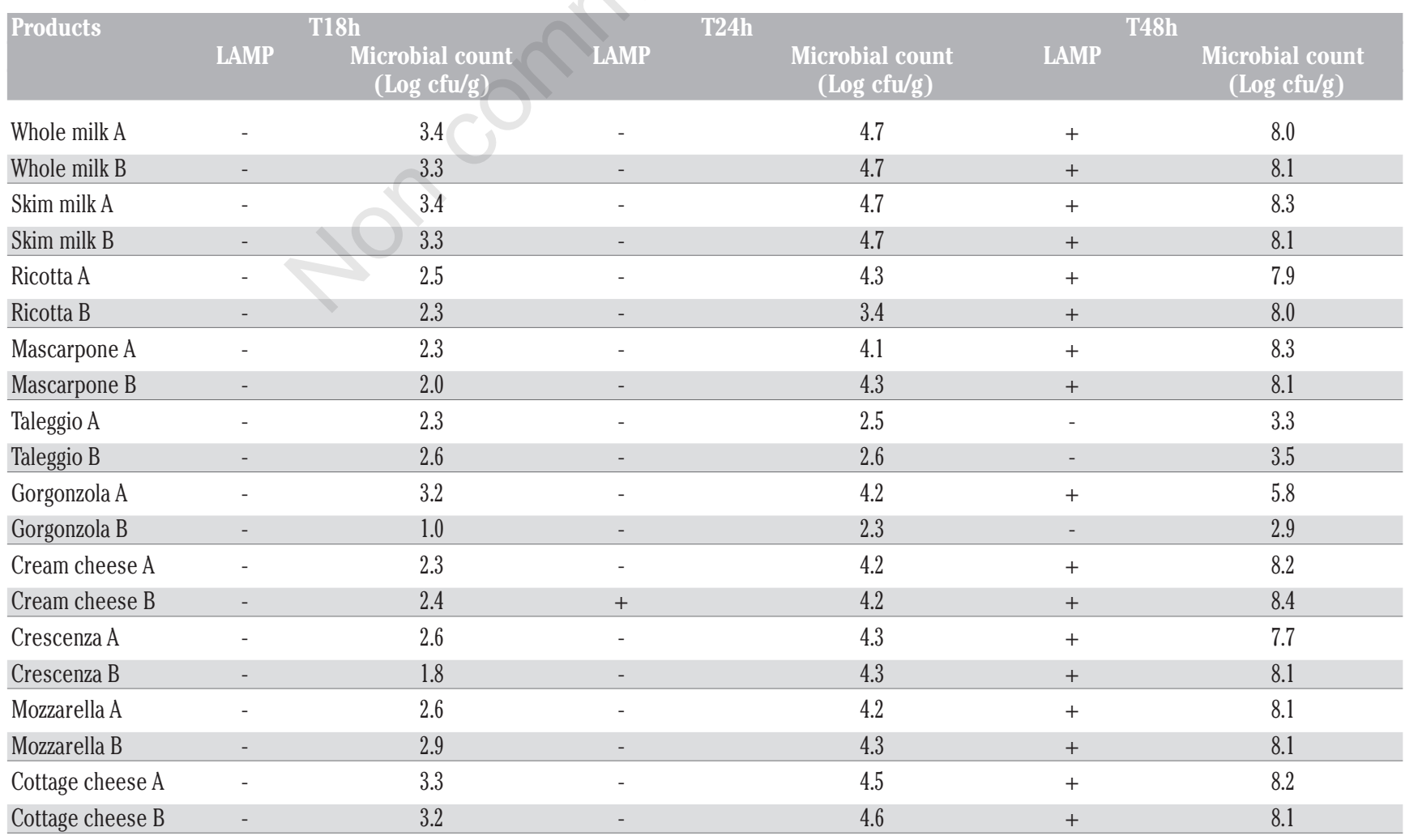

LAMP, loop-mediated isothermal amplification. 
nique to reveal the presence of L. monocytogenes, especially for official analyses of samples. The performance of culture-based analyses require at least 24-48 hours for the enrichment stage, with the addition of subsequent 24-48 hours for the growth of colonies in selective agar plate and of the time required to identification (e.g. biochemical) tests. Also PCR and real-time PCR-based analyses have been developed and used in the last years with good performances; nevertheless, some weaknesses were noticed in terms of time consumption for post detection procedures, high risk of cross-contamination and detection limit; even real-time PCR was used but some other disadvantages were noticed in terms of necessity of trained personnel and expensive equipment and reagents. Differently from these techniques, LAMP assay did not need complex pre-treatment and had very strong operability, easy-functionality and could be easily disseminated.

\section{Conclusions}

The LAMP assay tested in the present study was a specific and rapid method for detecting L. monocytogenes in broth cultures and dairy products. The preliminary data obtained should be confirmed with a validation to warrant constant good performances in terms of data quality obtained, time of analyses and standardisation of operative protocol. More studies are needed, not only to confirm the results obtained for dairy products, but also to extend the application of the method to other food of animal origin, including meat and meat products, fish and fish products and multicomposed food (e.g. deli foods).

\section{References}

Cho A-R, Dong H-J, Seo K-H, Cho S, 2014. Development of a loop-mediated isothermal amplification assay for detecting Listeria monocytogenes prfA in milk. Food Sci Biotechnol 23:467-74.
EFSA, 2015. The European Union Summary Report on Trends and Sources of Zoonoses, Zoonotic Agents and Food-borne Outbreaks in 2014. EFSA J 13:4329.

EFSA, 2016. The European Union Summary Report on Trends and Sources of Zoonoses, Zoonotic Agents and Food-borne Outbreaks in 2015. EFSA J 14:4634.

Hara-Kudo Y, Konishi N, Ohtsuka K, Hiramatsu R, Tanaka H, Konuma H, Takatori K, 2008. Detection of Verotoxigenic Escherichia coli O157 and $\mathrm{O} 26$ in food by plating methods and LAMP method: A collaborative study. Int J Food Microbiol 122:156-61.

Hara-Kudo Y, Yoshino M, Kojima T, Ikedo M, 2005. Loop-mediated isothermal amplification for the rapid detection of Salmonella. FEMS Microbiol Lett 253:155-61.

Kim D-H, Chon J-W, Kim H, Kim H-S, Choi D, Kim Y-J, Yim J-H, Moon J-S, Seo KH, 2014. Comparison of culture, conventional and real-time PCR methods for Listeria monocytogenes in foods. Korean J Food Sci Anim Resour 34:665-73.

Mori Y, Nagamine K, Tomita N, Notomi T, 2001. Detection of loop-mediated isothermal amplification reaction by turbidity derived from magnesium pyrophosphate formation. Biochem Biophys Res Commun 289:150-4.

Nagamine K, Hase T, Notomi T, 2002. Accelerated reaction by loop-mediated isothermal amplication using loop primers. Mol Cell Probes 16:223-9.

Notomi T, Okayama H, Masubuchi H, Yonekawa T, Watanabe K, Amino N, Hase T, 2000. Loop-mediated isothermal amplification of DNA. Nucleic Acids Res 28:E63.

Spanu C, Scarano C, Ibba M, Spanu V, De Santis EPL, 2015. Occurrence and traceability of Listeria monocytogenes strains isolated from sheep's milk cheese-making plants environment. Food Control 47:318-25.

Tang M-J, Zhou S, Zhang X-Y, Pu J-H, Ge Q-L, Tang X-J, Gao Y-S, 2011. Rapid and sensitive detection of Listeria monocytogenes by loop-mediated isothermal amplification. Curr Microbiol 63:511-6.

Tirloni E, Stella S, Drago S, Stampone G, Bernardi C, 2017. Loop-Mediated Isothermal Amplification (LAMP) for the detection of Anisakis simplex. LXX Convegno Sisvet, Napoli, June 28- July $1^{\text {st }} 2017$.

Unnerstad H, Bannerman E, Bille J, Danielsson-Tham ML, Waak E, Tham W, 1996. Prolonged contamination of a dairy with Listeria monocytogenes. Neth Milk Dairy J 50:493-9.

Walker SJ, Archer P, Banks JG, 1990. Growth of Listeria monocytogenes at refrigeration temperatures. J Appl Microbiol 68:157-62.

Wan C, Yang Y, Xu H, Aguilar ZP, Liu C, Lai W, Xiong Y, Xu F, Wei H, 2012. Development of a propidium monoazide treatment combined with loop-mediated isothermal amplification (PMA-LAMP) assay for rapid detection of viable Listeria monocytogenes. Int J Food Sci Technol 47:2460-7.

Wang D, Huo G, Ren D, Li X, 2010. Development and evaluation of a loop mediated isothermal amplification (LAMP) method for detecting Listeria monocytogenes in raw milk. J Food Safety 30:251-62.

Wang L, Li Y, Chu J, Xu Z, Zhong Q, 2012. Development and application of a simple loop-mediated isothermal amplification method on rapid detection of Listeria monocytogenes strains. Mol Biol Rep 39:445-9.

WHO-FAO, 2004. Risk assessment of Listeria monocytogenes in ready-to-eat foods. Available from: http://www.fao.org/docrep/010/y5394e/ y5394e00.htm

Zhang L, Pan ZM, Geng SZ, Chen X, Liu ZY, Zhao F, Jiao XA, 2012. A loopmediated isothermal amplification method targets the HisJ gene for the detection of foodborne Salmonella. Eur Food Res Technol 234:1055-62.

Zottola EA, Smith LB, 1991. Pathogens in cheese. Food Microbiol 8:171-82. 